\title{
Hemobilia Post Liver Biopsy: Mechanism, Presentation, Complications and Management
}

\author{
Norman Oneil Machado* \\ Department of Surgery, Sultan Qaboos University hospital, Muscat, OMAN
}

Received: September 09, 2015; Accepted: November 16, 2015; Published: November 28, 2015

*Corresponding author: Norman Oneil Machado, Department of Surgery, Sultan Qaboos University hospital, Muscat, OMAN, PO Box: 28, Postal code: 123, Tel: 0-96-8 99-432723; Email: oneilnorman@gmail.com

\begin{abstract}
Background: Liver biopsy is the gold standard for diagnosing various liver pathology. However, it may be associated with serious complications such as hemobilia, information of which is scarce in the literature.
\end{abstract}

Objective: This article intends to review various aspects of hemobilia including the mechanism, presentation, diagnosis and management.

Method: Literature review. A systematic review of the literature was performed by searching the PubMed and Medline databases, for all relevant articles in English on hemobilia, published from 1963 to June 2015.

Discussion: The search resulted in retrieval of 56 studies, involving 78 patients with hemobilia. The method of biopsy included percutaneous(51.8\%), ultrasound guided percutaneous biopsy(29.6\%), transjugular(11.1\%), laparoscopic biopsy(3.7\%). The predominant symptom of presentation was pain and GI bleed(42.2\%), Quincke's triad(39.4\%). The mean day of presentation was 5 days. The investigations carried out included angiogram(68\%),LFT(47.9\%), ultrasound abdomen(45.2\%), ERCP(24.6\%), endoscopy(16.4\%). The vascular anomaly contributing to hemobilia were arteriobiliary fistula(32.6\%), arterioportal fistula(36.9\%), pseudoaneurysm(21.7\%) and arteriovenous biliary fistula(trifistula) (4.3\%). Transarterial embolization was carried out in $61.2 \%$ of patients with $80 \%$ success in arresting bleeding. ERCP with clot extraction was carried out in $20.9 \%$. Surgical intervention included cholecystectomy (23\%), CBD exploration and clot extraction (9.23\%) and hepatic artery ligation (right or left branch)(9.23\%). The complications observed were pancreatitis (41.8\%), hemocholecystitis(21.8\%), cholangitis(10.9\%). Death was seen in $3.6 \%$ of patients.

Conclusion: Hemobilia is a rare complication post liver biopsy Angiogram is both diagnostic and therapeutic. Surgical interventions is limited to those who fail to respond to arterial embolization or develop complications in the gall bladder or CBD.

Keywords: Hemobilia; Liver biopsy; Transarterial; Embolization

\section{Introduction}

Hemobilia refers to bleeding into the biliary system. Several reports indicate its occurrence post liver biopsy [1-63]. Hemobilia was first described by Glisson in 1654, in a postmortem diagnosis of a young adult, stabbed by a sword in the liver [46]. Sandblom in 1948 used the term hemobilia to describe hemorrhage into the biliary tree, secondary to trauma [49].It was first reported as a complication of liver biopsy in 1967 [44]. In one of the largest multicenter study, reporting on the complications of liver biopsy, hemobilia was noted in 4 of 68,276 patients $(0.006 \%)$ [50]. In another report, the incidence of hemobilia, post percutaneous liver biopsy was reported to be $0.023 \%$ among 12,750 patients, accounting to $11.5 \%$ of all major complications [39]. However, several recent reports indicate the incidence of hemobilia post liver biopsy to be higher at just below $1 \%$ [20] and include $0.70 \%$ $(1 / 142)$ [47] and $(0.1 \%)$ [5, 7]. In this article the literature is reviewed and various factors that influence its cause and its management has been discussed.

\section{Methods}

Literature review. A systematic review of the literature was performed on hemobilia, by searching the PubMed and Medline databases for all relevant articles in English, published from 1963 to June 2015. The articles were retrieved using the MeSH(Medical Subject Headings) terms "hemobilia" and "liver biopsy". The data was extracted and studied for demographic details, method of liver biopsy, primary liver disorder warranting a biopsy, clinical presentation, the time of presentation, complications and management including radiological, endoscopic and surgical.

\section{Results}

The search resulted in retrieval of 56 studies involving 78 patients and majority (88\%) of these studies were case reports; seven of them were case series with 2 or more cases. Unfortunately, some of the studies lacked data regarding various parameters studied and hence the statistics had to be derived from data available (DA). Table 1 and Table 2. There were 21 male and 27 female patients (DA=48 patients) with an average age of 43.9 years (range 1.7-75 years). The biopsy methods included percutaneous biopsy (51.8\%), ultrasound guided percutaneous biopsy (29.6\%), transjugular biopsy (11.1\%), laparoscopic biopsy (3.7\%) and laparotomy and biopsy (1.85\%). The predominant symptom of presentation was pain and GI bleed (42.2\%), Quincke's triad (39.4\%), pain and jaundice (5.6\%), GI 
Table 1: Demographic Details, Presentation, Complications.

\begin{tabular}{|c|c|c|c|c|c|c|}
\hline & $\begin{array}{l}\text { No } \\
\text { Of pts }\end{array}$ & $\begin{array}{l}\text { Age- in } \\
\text { yrs } \\
\text { Sex }\end{array}$ & $\begin{array}{l}\text { Liver Biopsy } \\
\text { Technique }\end{array}$ & $\begin{array}{l}\text { Presentation } \\
\text { Pain } \\
\text { Jaundice } \\
\text { GI bleed }\end{array}$ & \begin{tabular}{|c|} 
Day- of presentation \\
Post \\
procedure
\end{tabular} & $\begin{array}{l}\text { Complications } \\
\text { Cholecystitis } \\
\text { Cholangitis } \\
\text { Pancreatitis }\end{array}$ \\
\hline Zhou HB 2014 & 1 & $\begin{array}{c}57 \\
\mathrm{~F}\end{array}$ & USbps & $\begin{array}{c}\mathrm{P} \\
\text { GiBl-mel }\end{array}$ & 7 & $\begin{array}{c}\text { Cholecystitis } \\
\text { Cholangitis } \\
\text { Pancreatitis } \\
\text { Stress gastric ulcers }\end{array}$ \\
\hline Qureshi $^{2} 2014$ & 1 & $\begin{array}{l}29 \\
F\end{array}$ & Pbps & QT & 5 & NA \\
\hline $\begin{array}{c}\text { Zaleska-Dorobisz }^{3} \\
2014\end{array}$ & 1 & $\begin{array}{l}10 \\
M\end{array}$ & USbps & $\begin{array}{c}\mathrm{P} \\
\text { GiBl-Mel/hem }\end{array}$ & 7 & Nil \\
\hline $\begin{array}{l}\text { Howlett } \mathrm{DC}^{7}-2013 \\
\text { (UK audit) }\end{array}$ & 2/ 3455bpsy & NA & NA & $\begin{array}{c}\mathrm{P} \\
\text { GI-Bl Mel }=2\end{array}$ & NA & NA \\
\hline Plerhopies TA 2013 & 1 & $\begin{array}{l}69 \\
F\end{array}$ & TJbps & QT & $<1$ & $\begin{array}{c}\text { Cholecystits, gall bladder } \\
\text { rupture, } \\
\text { Intra-abdominal fluid collection }\end{array}$ \\
\hline Marynissen $\mathrm{T}^{5} 2012$ & $6 / 12 \mathrm{HB}$ & NA & NA & QT-6 & 6 & NA \\
\hline Gandhi $V^{6} 2011$ & $1 / 22(\mathrm{HB})$ & NA & USbps & NA & 6 & NA \\
\hline Kawakubo K 2011 & 1 & $\begin{array}{l}67 \\
M\end{array}$ & EUS-bps & $\begin{array}{l}\mathrm{P} \\
\mathrm{J}\end{array}$ & 4 & Nil \\
\hline Egritas $0^{9} 2010$ & 1 & $\begin{array}{l}7 \\
\mathrm{~F}\end{array}$ & USbps & $\begin{array}{c}\mathrm{P} \\
\text { Gibl- Hem\& Mel }\end{array}$ & 2 & $\begin{array}{l}\text { Cholecystitis } \\
\text { pancreatitis }\end{array}$ \\
\hline Koshy CG ${ }^{11} 2010$ & 1 & $\begin{array}{l}36 \\
F\end{array}$ & Tjbps & $\begin{array}{l}\text { Hypotension- } 4 \mathrm{hr} \\
\text { after procedure }\end{array}$ & $\begin{array}{l}<1 \\
4 \mathrm{hr}\end{array}$ & $\begin{array}{l}\text { Intra-peritoneal bleeding } \\
\text { from subcapsular venous } \\
\text { pseudoaneurysm }\end{array}$ \\
\hline Hendriks $\mathrm{M}^{10} 2009$ & 1 & $\begin{array}{l}42 \\
F\end{array}$ & USbps & $\begin{array}{c}\mathrm{P} \\
\text { Gibld-hem }\end{array}$ & 4 & $\begin{array}{c}\text { Pancreatitis } \\
\text { CBD obstruction }\end{array}$ \\
\hline Peña LR ${ }^{12} 2009$ & 1 & $\begin{array}{l}56 \\
M\end{array}$ & Pbps & $\begin{array}{l}\mathrm{P} \\
\mathrm{J}\end{array}$ & 7 & $\begin{array}{c}\text { Pancreatitis } \\
\text { Reaccumulation of clot after } \\
\text { initial extraction. repeat ERCP } \\
\text { 3days and stenting }\end{array}$ \\
\hline Wood B 132009 & 1 & $\begin{array}{l}11 \\
M\end{array}$ & USbps & $\begin{array}{c}\mathrm{P} \\
\text { GiBl- Mel }\end{array}$ & 14 & Pancreatitis \\
\hline Li F ${ }^{14} 2009$ & 1 & $\begin{array}{l}51 \\
M\end{array}$ & USbps & $\begin{array}{c}\mathrm{P} \\
\text { GiBl- Mel. }\end{array}$ & $1 \mathrm{hr}$ & Pancreatitis \\
\hline Prata $\mathrm{F}^{15} 2008$ & 2 & $\begin{array}{l}45 \& 37 \\
F \& M\end{array}$ & 2-USbps & QT-2 & $5 \& 4$ & cholangitis \\
\hline Rogart JN ${ }^{16} 2008$ & 1 & $\begin{array}{c}58 \\
\mathrm{~F}\end{array}$ & Tjbps & $\begin{array}{c}\mathrm{P} \\
\text { Gi-bl- Mel }\end{array}$ & 2 & Pancreatitis \\
\hline Gurakuqi GC ${ }^{17} 2008$ & 1 & $\begin{array}{l}60 \\
M\end{array}$ & $\begin{array}{c}\text { TJbps } \\
\text { (advanced liver } \\
\text { disease) }\end{array}$ & $\begin{array}{c}\mathrm{P} \\
\text { Gi-Bl-Hem }\end{array}$ & 1 & Died-multiorgan failure \\
\hline Bergmann $\mathrm{OM}^{18} 2007$ & 1 & $\begin{array}{c}55 \\
\mathrm{~F}\end{array}$ & Tjbps & $\begin{array}{c}J \\
\text { Confusion }\end{array}$ & 2 & $\begin{array}{c}\text { Bilhemia with deteriorating LFT } \\
\text { Acute pancreatitis } \\
\text { ARDS }\end{array}$ \\
\hline Dallal $\mathrm{RM}^{19} 2007$ & 1 & $\begin{array}{l}34 \\
F\end{array}$ & $\begin{array}{l}\text { Laparoscbps- } \\
\text { during bypass } \\
\text { surgery for } \\
\text { obesity }\end{array}$ & $P$ & 1 & Nil \\
\hline Edden $Y^{20} 2006$ & 1 & $\begin{array}{l}15 \\
M\end{array}$ & Pbpsy & $P$ & 12 & $\begin{array}{l}\text { Cholecystitis. Persistent fullness } \\
\text { of GB with blood- } 14 \text { days }\end{array}$ \\
\hline Albuquerque $\mathrm{W}^{21} 2005$ & 1 & $\begin{array}{c}21 \\
F\end{array}$ & NA & QT & $1 \mathrm{hr}$ & $\begin{array}{l}\text { Pancreatitis, } \\
\text { Cholecystitis . }\end{array}$ \\
\hline Nowak $A^{23} 2005$ & 1 & $55 \mathrm{~F}$ & Pbps & $\mathrm{P}$ & 4 & Pancreatitis \\
\hline
\end{tabular}




\begin{tabular}{|c|c|c|c|c|c|c|}
\hline Lin $\mathrm{CL}^{24} 2005$ & 1 & $\begin{array}{l}68 \\
F\end{array}$ & USpbs & $P$ & 2 & $\begin{array}{l}\text { Recurrence of bleeding after } \\
\text { discharge following ERCP- } \\
\text { embolization after } 2 \text { days }\end{array}$ \\
\hline Kruse-Jarres 222005 & 1 & $\begin{array}{l}39 \\
\mathrm{M}\end{array}$ & Tjbps & $\begin{array}{c}\text { P } \\
\text { LFT-abnormal }\end{array}$ & 4 & nil \\
\hline Hodgson $\mathrm{RS}^{25} 2004$ & 1 & $\begin{array}{l}63 \\
F\end{array}$ & USbps & NA & 4 & Pancreatitis \\
\hline Sharma R ${ }^{26} 2004$ & 1 & $55 \mathrm{M}$ & NA & QT & 3 & Nil \\
\hline Hashimoto $\mathrm{M}^{48} 2004$ & $\begin{array}{c}3 /(8 \text { cases of } \\
\text { HB })\end{array}$ & NA & USbps & $\begin{array}{c}\mathrm{P} \\
\text { Gibl-Mel } \\
-3 \text { Cases } \\
\end{array}$ & 3 & $\begin{array}{l}\text { Liver infarction post } \\
\text { embolisation }\end{array}$ \\
\hline $\operatorname{Rossi}^{47} 2002$ & $\begin{array}{l}1 / 142 \\
\text { (usbps) }\end{array}$ & $\begin{array}{c}53 \\
\mathrm{~F}\end{array}$ & USbps & $\begin{array}{c}\mathrm{P} \\
\text { Gi-Bl- Mel }\end{array}$ & 6 & NA \\
\hline Machicao VII 2002 & 1 & $\begin{array}{l}49 \\
M\end{array}$ & Pbps & $\begin{array}{c}\mathrm{P} \\
\text { Gi-bleed }\end{array}$ & 10 & Pancreatitis \\
\hline Sood A. ${ }^{52} 2002$ & 1 & $55 \mathrm{M}$ & Pbps & QT & NA & Pancreatitis \\
\hline Asselah T $\mathrm{T}^{27} 2001$ & 1 & $43 \mathrm{~F}$ & Pbps & P Gibl-Mel & 4 & Pancreatitis \\
\hline Coelho JC ${ }^{28} 2001$ & 1 & $47 \mathrm{M}$ & Pbps & P Gi-bl-Mel & 2 & Cholecystitis \\
\hline Gomez-Valero ${ }^{29} 2001$ & 1 & $53 \mathrm{~F}$ & Pbps & P Gibl-Mel & 4 & Pancreatitis \\
\hline Gama-Odrigues $]^{30} 2001$ & 2 & NA & Pbps & P Gibl-2 Cases & 5 & NA \\
\hline Moehler M M $^{32} 2000$ & 1 & $43 \mathrm{~F}$ & Mini-laparcBps & $\mathrm{P}$ & 4 & NA \\
\hline Eurvilaichit C ${ }^{33} 1999$ & 1 & $35 \mathrm{~F}$ & Pbps & P Gi-bl- mel & 3 & NA \\
\hline Jornod P $\mathrm{P}^{34} 1999$ & 1 & $75 \mathrm{~F}$ & Pbps & P J & 8 & Pancreatitis \\
\hline Lee SL ${ }^{35} 1999$ & 1 & $30 \mathrm{~F}$ & Pbps & P Gi-bl-Hem\&Mel & 2 & $\begin{array}{c}\text { Necrotic GB-cystic duct blocked } \\
\text { with clot }\end{array}$ \\
\hline $\begin{array}{c}\mathrm{Kim} \mathrm{HJ}^{36} \\
1999\end{array}$ & 1 & NA & NA & NA & NA & Cholecystitis / Pancreatitis \\
\hline $\begin{array}{l}\text { Kwauk ST }^{37} \\
1998\end{array}$ & 1 & $\begin{array}{c}35 \\
\mathrm{~F}\end{array}$ & Pbps & $\begin{array}{c}\text { Gibl-Mel } \\
\text { J-2 weeks later after } \\
\text { lap cholecystectomy }\end{array}$ & 10 & $\begin{array}{l}\text { nil } \\
\text { Acalculouscholecystitis }\end{array}$ \\
\hline $\begin{array}{c}\text { Richardson } \mathrm{SC}^{38} \\
1998\end{array}$ & 1 & $\begin{array}{l}49 \\
M\end{array}$ & USbps & $\begin{array}{c}\text { P } \\
\text { GIbl-Mel }\end{array}$ & 9 & Pancreatitis \\
\hline $\begin{array}{c}\text { Dousset B } 39 \\
1997\end{array}$ & 11 & NA & Pbps & $\begin{array}{l}\text { QT- } 7 \\
4=\mathrm{NA}\end{array}$ & $\begin{array}{l}\text { Median-3 } \\
(1-25)\end{array}$ & $\begin{array}{l}\text { Pancreatitis-32\%- (4 pt) } \\
\text { Cholangitis-37\% (4 pt) }\end{array}$ \\
\hline Cacho $G^{45} 1996$ & 1 & NA & Pbps & P GI-bld- mel & 3 & Cholecystitis \\
\hline Grieco $A^{53} 1996$ & 1 & $65 \mathrm{~F}$ & Pbps & QT & $18 \mathrm{hr}$ & Nil \\
\hline Van Os EC ${ }^{54} 1996$ & 1 & $64 \mathrm{M}$ & Pbps & $\begin{array}{c}\text { P Gi- bl- } \\
\text { hematochezia }\end{array}$ & $24 \mathrm{hr}$ & Pancreatitis \\
\hline $\begin{array}{l}\text { Jabbour } N^{55} \\
1995\end{array}$ & 3 & $\begin{array}{l}26,64,1.7 \\
M=2, F=1\end{array}$ & $\begin{array}{c}\text { Pbps }=1 \\
\text { Laprbps }=1 \\
\text { Usbps }=1\end{array}$ & $\begin{array}{l}1 \text { pt-Asymptomatic- } \\
\text { detected during } \\
\text { routine angiogram } \\
\text { post transplant } \\
2^{\text {nd }} \text { pt-J+ Gibld } \\
3 \mathrm{rdpt}=\text { abnormal } \\
\text { LFT=hepatic } \\
\text { angiogram detected }\end{array}$ & NA & $\begin{array}{l}\text { Multiorgan failure-1 } \\
\text { Liver infarction-1 }\end{array}$ \\
\hline $\begin{array}{l}\text { De Ribot } X^{56} \\
1995\end{array}$ & 1 & $\begin{array}{c}55 \\
\mathrm{~F}\end{array}$ & Pbps & $\begin{array}{c}\text { P } \\
\text { GIbl-Mel }\end{array}$ & 1 & Pancreatitis \\
\hline $\begin{array}{l}\text { Figueras J }^{57} \\
1994\end{array}$ & 1 & $\begin{array}{l}7 \\
\mathrm{~F}\end{array}$ & Pbps & $\begin{array}{c}\mathrm{P} \\
\text { Gi-bl-Mel }\end{array}$ & $24 \mathrm{hr}$ & $\begin{array}{c}\text { Died of massive bleeding } \\
\text { despite TAE and before surgical } \\
\text { intervention }\end{array}$ \\
\hline $\begin{array}{c}\text { Merhav }^{58} \\
1993\end{array}$ & 1 & $\begin{array}{l}41 \\
\mathrm{M}\end{array}$ & Pbps & $\begin{array}{c}\mathrm{P} \\
\text { Gi-bl-Mel }\end{array}$ & 1 & NA \\
\hline $\begin{array}{c}\text { Manzarbietia }^{59} \\
1993\end{array}$ & 1 & $\begin{array}{l}51 \\
M\end{array}$ & USbps & $\begin{array}{c}\mathrm{P} \\
\text { Gi-bl-Mel }\end{array}$ & 3 & Pancreatitis \\
\hline
\end{tabular}




\begin{tabular}{|c|c|c|c|c|c|c|}
\hline $\begin{array}{c}\text { Lichtenstein DR } \\
1992^{40}\end{array}$ & 1 & $\begin{array}{l}39 \\
M\end{array}$ & Pbps & $\begin{array}{l}\mathrm{P} \\
\text { Gi-bl- mel-48 hrs } \\
\text { later }\end{array}$ & 4 & NA \\
\hline $\begin{array}{c}\text { Okazaki M } \\
1991^{60}\end{array}$ & $1 / 10 \mathrm{HB}$ & NA & Pbps & $\begin{array}{c}\mathrm{P} \\
\text { Gi-bl-Mel-Hem }\end{array}$ & 2 & NA \\
\hline $\begin{array}{c}\text { Attiyeh FF }{ }^{41} \\
1976\end{array}$ & 1 & $\begin{array}{l}16 \\
M\end{array}$ & Pbps & QT & 3 & NA \\
\hline $\begin{array}{c}\text { Ball TJ }^{42} \\
1975\end{array}$ & 1 & & Pbps & QT & 2 & NA \\
\hline $\begin{array}{c}\text { Levinson JD }{ }^{43} \\
1972\end{array}$ & 1 & $\begin{array}{l}40 \\
\mathrm{M}\end{array}$ & Pbps & QT & 2 & NA \\
\hline $\begin{array}{c}\text { Cox } \mathrm{EF}^{44} \\
1967\end{array}$ & 1 & $\begin{array}{l}27 \\
F\end{array}$ & Pbps & QT & NA & NA \\
\hline & 78 & $\begin{array}{c}\mathrm{M}=21 \\
\mathrm{~F}=27 \\
\text { Age- Avg- } \\
43.9 \\
\text { Range-1.7 } \\
\text { to } 75 \\
\\
\\
\\
\text { DA=48 } \\
\text { cases }\end{array}$ & $\begin{array}{l}\text { Pbps=28 } \\
\text { Usbps-16 } \\
\text { Tjbps- } 6 \\
\text { Lapcbps=2 } \\
\text { Laprbps }=1 \\
\text { EUSbps }=1\end{array}$ & $\begin{array}{c}\mathrm{QT}=25 \\
\mathrm{P}+\mathrm{Gibl}=30 \\
\mathrm{P}+\mathrm{J}=4 \\
\mathrm{RAPT}=2 \\
\text { Gibl=2 } \\
\mathrm{J}=1 \\
\text { Hypotension=1 } \\
\text { Confusion=1 } \\
\\
\text { DA =71 cases }\end{array}$ & Median -4 days & $\begin{array}{c}\text { Cholecystitis-12 }(21.81 \%) \\
\text { Pancreatitis-23 }(41.81 \%) \\
\text { Cholangitis- } 6(10.9 \%) \\
\text { Liver Infarction-2 }(3.6 \%) \\
\text { Mulitiorgan failure-2 }(3.6 \%) \\
\text { Bilhemia-1 }(1.81 \%) \\
\text { Death-2 }(3.6 \%) \\
\text { DA=55 cases }\end{array}$ \\
\hline
\end{tabular}

$\mathrm{HB}=$ haemobilia, USbps= ultrasound guided biopsy, Pbps=percutaneous biopsy, Tjbps= transjugular biopsy, EUS-bps= Endoscopic ultrasound guided biopsy, Lapcbps= laparoscopic biopsy, Laprbps= laparotomy and biopsy, DA= data available, P=pain, J= Jaundice, Gibl=GI bleed, Gibl-Mel= Gi bleed presenting as melena, GIbl-hem= Gi bleed presenting as hematemesis, QT=Quincke's triad, NA= not available, RAPT= routine angiogram post transplant

Table 2: Investigations, Management and Complications.

\begin{tabular}{|c|c|c|c|c|c|c|}
\hline $\begin{array}{l}\text { Series } \\
\text { Year }\end{array}$ & Investigations & $\begin{array}{l}\text { Nature of } \\
\text { fistula }\end{array}$ & $\begin{array}{l}\text { Management } \\
\text { (radiological/ } \\
\text { endoscopic) }\end{array}$ & $\begin{array}{l}\text { Surgical } \\
\text { Procedure }\end{array}$ & $\begin{array}{l}\text { Discharge } \\
\text { Day- } \\
\text { Post } \\
\text { procedure }\end{array}$ & $\begin{array}{l}\text { Hepatic disorder/ } \\
\text { co-morbidities }\end{array}$ \\
\hline $\begin{array}{l}\text { Zhou HB } \\
2014\end{array}$ & $\begin{array}{l}\text { LFT } \\
\text { US } \\
\text { MRCP } \\
\text { Endoscope } \\
\text { DSA }\end{array}$ & AVBF & $\begin{array}{l}\text { TAE- } \\
\text { Rt hepatic artery }\end{array}$ & Nil & 12 days & $\begin{array}{l}\text { Abnormal liver } \\
\text { function }\end{array}$ \\
\hline $\begin{array}{l}\text { Qureshi² } \\
2014\end{array}$ & $\begin{array}{l}\text { LFT } \\
\text { Angiogram } \\
\text { Endoscope }\end{array}$ & $\mathrm{ABF}$ & $\begin{array}{l}\text { TAE } \\
\text { ERCP- ClExt }\end{array}$ & Nil & NA & Nil \\
\hline $\begin{array}{l}\text { Zaleska-Dorobisz }{ }^{3} \\
2014\end{array}$ & $\begin{array}{l}\text { LFT } \\
\text { US } \\
\text { Endoscopy } \\
\text { Angiogram }\end{array}$ & $\begin{array}{l}\text { ABF } \\
\text { APF }\end{array}$ & $\begin{array}{l}\text { TAE- rt hepatic artery- } \\
\text { GianturooWallaoh coil }\end{array}$ & Nil & 4 & Chronic hepatitis B \\
\hline $\begin{array}{l}\text { Howlett DC } \\
\text { - } 2013 \\
\text { (UK audit) }\end{array}$ & NA & NA-2 & $\mathrm{NA}=2$ & $\mathrm{NA}=2$ & NA & NA \\
\hline $\begin{array}{l}\text { Plerhopies TA } \\
2013\end{array}$ & $\begin{array}{l}\text { LFT } \\
\text { CT } \\
\text { ERCP }\end{array}$ & NA & $\begin{array}{l}\text { ERCP-ClExt/stenting } \\
\text { Perc-chol }\end{array}$ & Nil & 3 & $\begin{array}{l}\text { Portal hypertension/ } \\
\text { deteriorating LFT/ } \\
\text { Hemosiderosis/ ESRD }\end{array}$ \\
\hline $\begin{array}{l}\text { Marynissen } T^{5} \\
2012\end{array}$ & $\begin{array}{l}\text { LFT } \\
\text { CT-2 } \\
\text { US-4 } \\
\text { Angiogram-6 }\end{array}$ & NA- 6 & TAE-6 & NA & NA & NA \\
\hline
\end{tabular}




\begin{tabular}{|c|c|c|c|c|c|c|}
\hline $\begin{array}{l}\text { Gandhi V6 } \\
2011\end{array}$ & $\begin{array}{l}\text { LFT } \\
\text { CT } \\
\text { Angiogram }\end{array}$ & NA & NA & NA & NA & NA \\
\hline $\begin{array}{l}\text { Kawakubo K } \\
2011\end{array}$ & $\begin{array}{l}\text { LFT } \\
\text { CT } \\
\text { Endoscope }\end{array}$ & NA & Nasobiliary drainage & Nil & 8 & Nil \\
\hline $\begin{array}{l}\text { Egritas } 0^{9} \\
2010\end{array}$ & $\begin{array}{l}\text { LFT } \\
\text { CT } \\
\text { Endoscope }\end{array}$ & NA & $\begin{array}{l}\text { Supportive measures- } \\
\text { blood products/iv } \\
\text { fluids }\end{array}$ & Nil & 10 & $\begin{array}{l}\text { Mitochondrial } \\
\text { disease, familial } \\
\text { intrahepatic } \\
\text { cholestasis }\end{array}$ \\
\hline $\begin{array}{l}\text { Koshy CG }{ }^{11} \\
2010\end{array}$ & $\begin{array}{l}\text { Endoscope } \\
\text { Hepatic Angiogram } \\
\text { Hepatic venogram }\end{array}$ & $\begin{array}{l}\text { APF } \\
\text { PsdA }\end{array}$ & $\begin{array}{l}\text { Supportive measures } \\
\text { TAE } \\
\text { Hepatic vein } \\
\text { embolization-platinum } \\
\text { coils }\end{array}$ & Nil & Few days & $\begin{array}{l}\text { Cryptogenic liver } \\
\text { cirrhosis, portal } \\
\text { hypertension, } \\
\text { moderate ascites }\end{array}$ \\
\hline $\begin{array}{l}\text { Hendricks MP }{ }^{10} \\
2009\end{array}$ & $\begin{array}{l}\text { US } \\
\text { ERCP-elongated opacities- } \\
\text { blood clots }\end{array}$ & NA & $\begin{array}{l}\text { ERCP - } \\
\text { sphincterotomy+ stent }\end{array}$ & Nil & NA & $\begin{array}{l}\text { Psoriasis } \\
\text { Liver biopsy to rule } \\
\text { out methotrexate } \\
\text { induced hepatitis }\end{array}$ \\
\hline $\begin{array}{l}\text { Peña } L^{12} \\
2009\end{array}$ & $\begin{array}{l}\text { LFT } \\
\text { MRCP } \\
\text { ERCP }\end{array}$ & NA & $\begin{array}{l}\text { ERCP-spinterotomy+ } \\
\text { ClExt-balloon +stenting }\end{array}$ & Nil & NA & Chronic hepatitis C \\
\hline $\begin{array}{l}\text { Wood } B^{13} \\
2009\end{array}$ & $\begin{array}{l}\text { LFT } \\
\text { Endoscopy } \\
\text { CT } \\
\text { Angiogram }\end{array}$ & $\begin{array}{l}\text { ABF } \\
\text { PsdA }\end{array}$ & NA & NA & NA & NASH \\
\hline $\begin{array}{l}\mathrm{Li} \mathrm{F}^{14} \\
2009\end{array}$ & $\begin{array}{l}\text { LFT } \\
\text { CT } \\
\text { MRCP }\end{array}$ & NA & $\begin{array}{l}\text { Supportive measures } \\
\text { only }\end{array}$ & Nil & 2 & $\begin{array}{l}\text { Alcoholic cirrhosis } \\
\text { Post liver / kidney } \\
\text { transplant }\end{array}$ \\
\hline $\begin{array}{l}\text { Prata } \mathrm{F}^{15} \\
2008\end{array}$ & $\begin{array}{l}\text { LFT-2 } \\
\text { US-1 } \\
\text { CT-1 } \\
\text { ERCP-2 }\end{array}$ & NA-2 & $\begin{array}{l}\text { ERCP-sphincterotomy } \\
+ \text { +ClExt }-2\end{array}$ & Nil & NA & Cirrhosis \\
\hline $\begin{array}{l}\text { Rogart JN }{ }^{16} \\
2008\end{array}$ & $\begin{array}{l}\text { LFT } \\
\text { CT } \\
\text { Endoscope } \\
\text { Angiogram }\end{array}$ & $\mathrm{ABF}$ & TAE & Nil & NA & Hepatitis C infection \\
\hline $\begin{array}{l}\text { Gurakuqi GC }{ }^{17} \\
2008\end{array}$ & $\begin{array}{l}\text { LFT } \\
\text { Endoscopy } \\
\text { Angiogram }\end{array}$ & $\mathrm{ABF}$ & TAE & Nil & died & $\begin{array}{l}\text { Alcoholic cirrhosis } \\
\text { Severe coagulopathy }\end{array}$ \\
\hline $\begin{array}{l}\text { Bergmann } \mathrm{OM}^{18} \\
2007\end{array}$ & $\begin{array}{l}\text { LFT } \\
\text { US } \\
\text { ERCP } \\
\text { Angiogram }\end{array}$ & $\begin{array}{l}\text { AVBF } \\
\text { (trifistula) }\end{array}$ & $\begin{array}{l}\text { TAE } \\
\text { ERCP-ClExt-stenting }\end{array}$ & Nil & 5 & $\begin{array}{l}\text { ESRD } \\
\text { Malignant melanoma }\end{array}$ \\
\hline $\begin{array}{l}\text { Dallal RM }{ }^{19} \\
2007\end{array}$ & $\begin{array}{l}\text { LFT } \\
\text { US } \\
\text { HIDA scan } \\
\text { ERCP }\end{array}$ & NA & NA & $\begin{array}{l}\text { Lap chol } \\
\text { CBD exploration }\end{array}$ & NA & $\begin{array}{l}\text { Hypertension, gastro- } \\
\text { oesophageal reflux, } \\
\text { polycystic ovarian } \\
\text { syndrome }\end{array}$ \\
\hline $\begin{array}{l}\text { Edden } Y^{20} \\
2006\end{array}$ & $\begin{array}{l}\text { US } \\
\text { On table cholangiogram }\end{array}$ & NA & Supportive measures & $\begin{array}{l}\text { Lap chol } \\
\text { and on table } \\
\text { cholangiogram }\end{array}$ & 2 & $\begin{array}{l}\text { Persistent elevated } \\
\text { liver enzymes/ } \\
\text { cryptogenic cirrhosis }\end{array}$ \\
\hline $\begin{array}{l}\text { Albuquerque } \mathrm{W}^{21} \\
2005\end{array}$ & $\begin{array}{l}\text { US-free fluid in peritoneal } \\
\text { cavity. GB- echogenic } \\
\text { material } \\
\text { ERCP+MRCP- edematous } \\
\text { pancreatitis/dilated GB,CBD } \\
\text { clots } \\
\text { Angiogram- no further } \\
\text { bleeding seen }\end{array}$ & NA & ERCP- ClExt -balloon & $\begin{array}{l}\text { Lap chol- } 5 \text { days } \\
\text { after ERCP for } \\
\text { recurrent abd } \\
\text { pain }\end{array}$ & NA & $\begin{array}{l}\text { Biopsy- preop for } \\
\text { renal transplant } \\
\text { protocol. } \\
\text { Hepatitis C }\end{array}$ \\
\hline
\end{tabular}




\begin{tabular}{|c|c|c|c|c|c|c|}
\hline $\begin{array}{l}\text { Kruse-Jarres }{ }^{22} \\
2005\end{array}$ & $\begin{array}{l}\text { US } \\
\text { CT }\end{array}$ & NA & $\begin{array}{l}\text { Conservatively } \\
\text { with factor V111 } \\
\text { replacement only }\end{array}$ & Nil & 4 & $\begin{array}{l}\text { HIV } \\
\text { Hepatitis C } \\
\text { Congenital } \\
\text { hemophilia }\end{array}$ \\
\hline $\begin{array}{l}\text { Nowak } A^{23} \\
2005\end{array}$ & $\begin{array}{l}\text { Endoscope } \\
\text { ERCP }\end{array}$ & NA & $\begin{array}{l}\text { ERCP ClExt=dormia } \\
\text { basket-nasobiliary } \\
\text { tube-thrombin infusion }\end{array}$ & Nil & 2 & $\begin{array}{l}\text { Diabetic } \\
\text { Chronic elevation of } \\
\text { ALAT- non-alcoholic } \\
\text { steatohepatitis }\end{array}$ \\
\hline $\begin{array}{l}\operatorname{Lin} \mathrm{CL}^{24} \\
2005\end{array}$ & $\begin{array}{l}\text { US- Gb-polypoidal mass } \\
\text { ERCP-blood in ampulla- clot } \\
\text { in CBD }\end{array}$ & APBF & $\begin{array}{l}\text { ERCP-sphincterotomy- } \\
\text { CLExt, } \\
\text { TAE- with gel foam- } \\
\text { segment VII }\end{array}$ & Nil & 7 & Chronic C hepatitis \\
\hline $\begin{array}{l}\text { Hodgson RS } \\
2004\end{array}$ & $\begin{array}{l}\text { US-free fluid around GB } \\
\text { Angiogram- } \\
\text { pseudoaneurysm/AP biliary } \\
\text { fistula }\end{array}$ & $\begin{array}{l}\text { APBF } \\
\text { PsdA }\end{array}$ & $\begin{array}{l}\text { TAE- Titanium } \\
\text { microcoils }\end{array}$ & Nil & 4 & Crohn's disease \\
\hline $\begin{array}{l}\text { Sharma } \mathrm{R}^{26} \\
2004\end{array}$ & $\begin{array}{l}\text { LFT- abnormal } \\
\text { US- dilated GB, mild IHD } \\
\text { dilatation } \\
\text { CT-distended GB, IHD } \\
\text { dilatation, blood clot in CBD } \\
\text { Angiography-filling defect in } \\
\text { Rt lobe } \\
\text { MRI/MRCP- for follow up }\end{array}$ & NA & TAE & Nil & NA & Hepatitis B \\
\hline $\begin{array}{l}\text { Hashimoto } \mathrm{M}^{48} \\
2004\end{array}$ & $\begin{array}{l}\text { US } \\
\text { CT } \\
\text { Angiogram }\end{array}$ & $\begin{array}{l}\text { PsdA-2 } \\
\text { ABF-1 }\end{array}$ & TAE & Nil & NA & \\
\hline $\begin{array}{l}\text { Rossi }^{47} \\
2002\end{array}$ & $\begin{array}{l}\text { US } \\
\text { ERCP-Blood clot in CBD } \\
\text { Angiogram }\end{array}$ & $\mathrm{ABF}$ & TAE & Nil & 7 & Hepatitis C \\
\hline $\begin{array}{l}\text { Machicao } \mathrm{VI}^{51} \\
2002\end{array}$ & $\begin{array}{l}\text { LFT } \\
\text { CT } \\
\text { Angiogram }\end{array}$ & APF & TAE & Nil & NA & Hepatitis C \\
\hline $\begin{array}{l}\text { Sood } A^{52} \text {. } \\
2002\end{array}$ & $\begin{array}{l}\text { LFT } \\
\text { CT } \\
\text { ERCP }\end{array}$ & NA & $\begin{array}{l}\text { ERCP-sphincterotomy } \\
+ \text { +ClExt }\end{array}$ & Nil & NA & Hepatitis C \\
\hline $\begin{array}{l}\text { Asselah T }{ }^{27} \\
2001\end{array}$ & $\begin{array}{l}\text { LFT } \\
\text { US } \\
\text { MRCP- acute pancreatitis/ } \\
\text { CBD clot }\end{array}$ & NA & NA & NA & NA & \\
\hline $\begin{array}{l}\text { Coelho JC'28 } \\
2001\end{array}$ & $\begin{array}{l}\text { US-thick walled GB-blood clot } \\
\text { intraluminal }\end{array}$ & NA & NA & $\begin{array}{l}\text { Lap } \\
\text { cholecystectomy }\end{array}$ & & \\
\hline $\begin{array}{l}\text { Gomez-Valero }{ }^{29} \\
2001\end{array}$ & $\begin{array}{l}\text { US } \\
\text { Angiogram }\end{array}$ & APF & TAE & Nil & 3 & \\
\hline $\begin{array}{l}\text { Gama-Odrigues } \mathrm{J}^{30} \\
2001\end{array}$ & $\begin{array}{l}\text { US } \\
\text { Angiogram } \\
\text { Laparoscopy }\end{array}$ & $\mathrm{ABF}=2$ & $\begin{array}{l}\text { Conservative } \\
\text { measures }=2\end{array}$ & $\begin{array}{l}\text { Lap } \\
\text { cholecystectomy } \\
\text { and ligation of } \\
\text { hepatic artery- } 2 \\
\text { cases }\end{array}$ & NA & NA \\
\hline $\begin{array}{l}\text { Moehler M }{ }^{32} \\
2000\end{array}$ & $\begin{array}{l}\text { US } \\
\text { Angiogram }\end{array}$ & APF & TAE & Nil & 3 & Nil \\
\hline $\begin{array}{l}\text { Eurvilaichit } \mathrm{C}^{33} \\
1999\end{array}$ & $\begin{array}{l}\text { US } \\
\text { Angiogram }\end{array}$ & $\mathrm{ABF}$ & TAE-gelfoam & Nil & 3 & Nil \\
\hline $\begin{array}{l}\text { Jornod } \mathrm{P}^{34} \\
1999\end{array}$ & $\begin{array}{l}\text { LFT } \\
\text { US } \\
\text { ERCP }\end{array}$ & NA & $\begin{array}{l}\text { ERCP-sphincterotomy- } \\
\text { ClExt }\end{array}$ & Nil & 3 & $\begin{array}{l}\text { Primary biliary } \\
\text { cirrhosis }\end{array}$ \\
\hline
\end{tabular}




\begin{tabular}{|c|c|c|c|c|c|c|}
\hline $\begin{array}{l}\text { Lee SL }^{35} \\
1999\end{array}$ & $\begin{array}{l}\text { LFT-abnormal } \\
\text { Angiogram- no source of } \\
\text { bleed seen } \\
\text { RBC tagged scan-post } \\
\text { cholecystectomy(abnormal } \\
\text { LFT/persistent pain)-NAD } \\
\text { ERCP- clot in CBD }\end{array}$ & NAD & $\begin{array}{l}\text { ERCP-postop on } 5^{\text {th }} \text { day } \\
\text {-Sphincterotomy-ClExt }\end{array}$ & $\begin{array}{l}\text { Open } \\
\text { cholecystectomy- } \\
\text { on } 3^{\text {rd }} \text { day- } \\
\text { necrotic GB }\end{array}$ & NA & $\begin{array}{l}\text { Hepatitis C } \\
\text { Went home and } \\
\text { returned after } 72 \mathrm{hrs}\end{array}$ \\
\hline $\begin{array}{l}\mathrm{Kim} \mathrm{HJ} \mathrm{H}^{36} \\
1999\end{array}$ & NA & NA & NA & NA & NA & NA \\
\hline $\begin{array}{l}\text { Kwauk ST }{ }^{37} \\
1998\end{array}$ & $\begin{array}{l}\text { CT-hematoma rt lobe of liver } \\
\text { ERCP-normal CBD/pancreatic } \\
\text { duct } \\
\text { LFT- abnormal-2 weeks later } \\
\text { Angiogram-24 days after } \\
\text { initial presentation and lap } \\
\text { chole }\end{array}$ & PsdA & $\begin{array}{l}\text { TAE-coil } \\
\text { Percutaneous drainage } \\
\text { of gall bladder bed } \\
\text { collection }\end{array}$ & Nil & 11 & Biliary cirrhosis \\
\hline $\begin{array}{l}\text { Richardson } \mathrm{SC}^{38} \\
1998\end{array}$ & ERCP & APF & NA & NA & NA & Hepatitis C \\
\hline $\begin{array}{l}\text { Dousset B }{ }^{39} \\
1997\end{array}$ & $\begin{array}{l}\mathrm{LFT}=11 \\
\mathrm{US}=11 \\
\text { Angiogram-11 }\end{array}$ & $\begin{array}{l}\mathrm{APF}=4 \\
\mathrm{PsdA}=2 \\
\mathrm{ABF}==3 \\
\mathrm{NAD}=1 \\
\text { Vascular flask=1 }\end{array}$ & $\begin{array}{l}\text { TAE- } \text { successful }=7 \\
\text { Failed }=2 \\
\text { technical difficulty } \\
\text { Complicated }=1 \\
\text { (ischemiccholecystitis }\end{array}$ & & NA & NA \\
\hline $\begin{array}{l}\text { Cacho G }{ }^{45} \\
1996\end{array}$ & $\begin{array}{l}\text { US } \\
\text { Endoscopy } \\
\text { Angiogram }\end{array}$ & APF & TAE & Cholecystectomy & NA & nil \\
\hline $\begin{array}{l}\text { Grieco A }{ }^{53} \\
1996\end{array}$ & $\begin{array}{l}\text { US } \\
\text { Angiogram-AV fistula- } 3^{\text {rd }} \\
\text { liver segment } \\
\text { ERCP-clot }\end{array}$ & AVF & TAE- gelfoampledgets & Nil & NA & $\begin{array}{l}\text { Polyneuropathy/ } \\
\text { endocrinopathy/ } \\
\text { monoclonal } \\
\text { gammapathy }\end{array}$ \\
\hline $\begin{array}{l}\text { Van Os EC }{ }^{54} \\
1996\end{array}$ & $\begin{array}{l}\text { Angiogram } \\
\text { Endoscopy }\end{array}$ & PsdA & TAE & Nil & NA & Drug related hepatitis \\
\hline $\begin{array}{l}\text { Jabbour } \mathrm{N}^{55} \\
1995 \\
(3 \text { cases })\end{array}$ & $\begin{array}{l}\text { Doppler-2 } \\
\text { Angiogram-3 } \\
\text { Radionucleide scanning-1 } \\
\text { Endoscopy-1 }\end{array}$ & APF-3 & $\begin{array}{l}\text { Conservative-2 } \\
\text { TAE-1 }\end{array}$ & $\begin{array}{l}\text { CBD exploration, } \\
\text { and } 2^{\text {nd }} \text { liver } \\
\text { transplant for } \\
\text { infarction- } 1 \text { case }\end{array}$ & NA & $\begin{array}{l}\text { Non A/B hepatitis-1 } \\
\text { Chronic active } \\
\text { hepatititis-1 } \\
\text { Cat's eye syndrome-1 }\end{array}$ \\
\hline $\begin{array}{l}\text { deRibot X56 } \\
1995\end{array}$ & ERCP & NA & NA & NA & NA & $\begin{array}{l}\text { Chronic elevated liver } \\
\text { enzyme }\end{array}$ \\
\hline $\begin{array}{l}\text { Figueras J }{ }^{57} \\
1994\end{array}$ & Angiogram & $\mathrm{ABF}$ & TAE & Nil & Died & Post liver transplant \\
\hline $\begin{array}{l}\text { Merhav }^{58} \\
1993\end{array}$ & $\begin{array}{l}\text { US } \\
\text { Angiogram }\end{array}$ & PsdA & $\begin{array}{l}\text { Directpercutaneous } \\
\text { emoblisation-ultrasound } \\
\text { guided.(DPE) } \\
\text { TAE-technically was } \\
\text { not possible- tortuous } \\
\text { allograft hepatic artery }\end{array}$ & Nil & NA & Post liver transplant \\
\hline \begin{tabular}{|l} 
Manzarbietia ${ }^{59}$ \\
1993
\end{tabular} & $\begin{array}{l}\text { CT } \\
\text { MRCP }\end{array}$ & NA & NA & NA & NA & Post liver transplant \\
\hline $\begin{array}{l}\text { Lichtenstein DR } \\
1992^{40}\end{array}$ & $\begin{array}{l}\text { US } \\
\text { Angiogram }\end{array}$ & $\mathrm{ABF}$ & $\begin{array}{l}\text { TAE- gelfoam and } \\
\text { Gianturco coil }\end{array}$ & Nil & 3 & Nil \\
\hline \begin{tabular}{|l|} 
Okazaki M \\
1991 \\
\end{tabular} & $\begin{array}{l}\text { US } \\
\text { Angiogram }\end{array}$ & $\mathrm{ABF}$ & TAE-gelfoam & NA & NA & Nil \\
\hline $\begin{array}{l}\text { Attiyeh } \mathrm{FF}^{41} \\
1976\end{array}$ & $\begin{array}{l}\text { LFT } \\
\text { US }\end{array}$ & NA & $\begin{array}{l}\text { Supportive measures } \\
\text { only }\end{array}$ & $\begin{array}{l}\text { Cholecystectomy/ } \\
\text { CBD exploration/ } \\
\text { left hepatic artery } \\
\text { ligation }\end{array}$ & & Hodgkins disease \\
\hline
\end{tabular}




\begin{tabular}{|c|c|c|c|c|c|c|}
\hline $\begin{array}{l}\text { Ball TJ }{ }^{42} \\
1975\end{array}$ & $\begin{array}{l}\text { LFT } \\
\text { ERCP } \\
\text { Angiogram }\end{array}$ & NAD & NA & NA & NA & NA \\
\hline $\begin{array}{l}\text { Levinson JD }{ }^{43} \\
1972\end{array}$ & $\begin{array}{l}\text { LFT } \\
\text { Angiogram-linear collection } \\
\text { of contrast close to biopsy }\end{array}$ & NAD & ND & $\begin{array}{l}\text { T tube } \\
\text { decompression } \\
\text { of CBD } \\
\text { Left hepatic } \\
\text { artery branch } \\
\text { ligation }\end{array}$ & NA & $\begin{array}{l}\text { Micronodular } \\
\text { cirrhosis } \\
\text { Granulomatous liver } \\
\text { disease, Sarcoidosis }\end{array}$ \\
\hline $\begin{array}{l}\text { Cox EF } 44 \\
1967\end{array}$ & NA & NA & NA & NA & NA & NA \\
\hline & $\begin{array}{l}\text { Angiogram=50(68\%) } \\
\text { LFT=35 }(47.9 \%) \\
\text { US=33 }(45.2 \%) \\
\text { ERCP=18 }(24.6 \%) \\
\text { Endoscope }=12(16.4 \%) \\
\text { MRCP=6 }(8.21 \%) \\
\text { Radionucleide scan=3 }(4.1 \%)\end{array}$ & $\begin{array}{l}\mathrm{ABF}=15 \\
(32.6 \%) \\
\mathrm{APF}=16 \\
(16.9 \%) \\
\mathrm{PsdA}=10 \\
(21.7 \%) \\
\mathrm{AVBF}=2 \\
(4.34 \%) \\
\mathrm{NAD}=4 \\
(8.69 \%) \\
\\
\text { DA=46 }\end{array}$ & $\begin{array}{l}\text { TAE }=38(61.2 \%) \\
\text { ERCP+ClExt=13 } \\
(20.9 \%) \\
\text { Supportive measures } \\
\text { only=11 }(17.7 \%) \\
\text { DPE }=1(1.6 \%) \\
\text { Percchol=1(1.6\%) } \\
\text { NBD=1(1.6\%) } \\
\text { DA=62 }\end{array}$ & $\begin{array}{l}\text { NPP=30 (46.1\%) } \\
\text { Chcyst=15 } \\
(23.07 \%) \\
\text { CBDE=4 (6.15\%) } \\
\text { HAL=6 } \\
(9.23 \%) \\
\\
\\
\\
\text { DA=65 }\end{array}$ & $\begin{array}{l}\text { Median=6 } \\
\text { days }\end{array}$ & \\
\hline
\end{tabular}

bleed alone $(2.8 \%)$, and those detected on routine angiogram post liver transplant and liver biopsy (2.8\%) and one case each of confusion (1.96\%), hypotension and jaundice alone. The mean day of presentation was 5 days. The investigations that facilitated in diagnosis included angiogram (68\%), LFT (47.9\%), ultrasound (US) abdomen (45.2\%), ERCP (24.6\%), endoscopy (16.4\%), MRCP $(8.2 \%)$ and radio nucleide scan (4.1\%). Angiogram detected arteriobiliary fistula in $(32.6 \%)$, arterioportal fistula $(36.9 \%)$, pseudoaneurysm (21.7\%), arteriovenous biliary fistula-Trifistula (4.3\%) and no abnormality (8.69\%), among the 46 patients were data was available. Transarterial embolization was carried out in $61.2 \%$ and was successful in $80 \%$ of these cases in arresting the bleeding. ERCP with clot extraction was required in $20.9 \%$ of patients where CBD clot persisted. In $17.7 \%$ of patients no radiological or endoscopic measures were carried out and were managed with supportive therapy only. There was one patient each who underwent percutaneous cholecystostomy, nasobiliary drainage of the biliary tract and ultrasound guided direct percutaneous injection of the vascular fistula, due to technical difficulty in catheterisation and embolization of hepatic artery because of its tortuosity. No additional surgical procedures were carried out in $46.1 \%$; however among the remaining patients, $23 \%$ underwent cholecystectomy, 6.15\% underwent CBD exploration and clot removal and $9.23 \%$ underwent hepatic artery ligation. The complications noted were pancreatitis (41.8\%), cholecystitis (21.8\%), cholangitis (10.9\%), liver infarction in patients with liver transplant $(3.6 \%)$, multiorgan failure $(3.6 \%)$, bilhemia $(1.8 \%)$ among the 55 patients were data was available. Death was noted in 2 patients(3.6\%). The median stay of patients was
6 days. However in many of the patients with complications, the data of discharge was not available and the median day of stay of 6 days noted, is likely to represent those without complications.

\section{Mechanism}

The term hemobilia relates to bleeding into the biliary system. Majority of the bleeding are post liver biopsy, a reflection of the recentincrease in this invasive procedure, to establish diagnosis of liver pathology [62].They could also be secondary to gallstones, vascular malformations, parasitic infestation, inflammation, tumours, post cholecystectomy [6, 63], acute cholecystitis [64] and trauma [6]. Most of the bleeding is into the intra-hepatic biliary system [1-6, 9-22]. However, bleeding into extrahepatic bile ducts and gall bladder is also reported [8]. The vessels that could be involved include cystic artery, anomalous hepatic artery, portal vein andhepatic artery or its intrahepatic branches $[1,6]$.Due to the close proximity of the intrahepatic bile duct, hepatic artery and portal vein, a puncture needle during liver biopsy,could easily lead to injuryof all these structures to form arteriovenous bile duct fistula, arterial bile duct fistula and venous bile duct fistula [1]. Bleeding from venous bile duct fistula often stops spontaneously, because of low venous pressure [1]. The bleeding of venous origin could however lead to significant bleeding, if they bleed freely into peritoneal cavity through a subcapsular located bleeding site [11]. An arteriobiliary fistula on the other hand is likely to be symptomatic because of bleeding from a high pressure vascular system into low pressure biliary tract. Hemobilia could also be due to pseudoaneurysm, which was seen in $21 \%$ in this review. The pseuodaneurysm formation 
may be delayed after a biopsy and are usually a consequence hematoma, progressive compression, and chronic inflammation, all leading to low-grade damage within the liver parenchyma $[18,20,55]$. This could then predispose to aneurysmal changes in the adjoining hepatic arterial branch, forming a pseudoaneurysm [55]. The risk of vascularbiliary fistula is believed to be increased, when biopsies are carried out in the presence biliary duct obstruction and infection, as the hepatic arterial blood flow increases in such condition [1]. Moreover, hyperplasia and distension of the arterial peribiliary vascular plexus and the feeding hepatic artery branch in periportal area enhances this risk [1]. Fistula involving the hepatic artery, hepatic vein and biliary tree could cause an intrahepatic trifistula leading to bilhemia and Hemobilia [18]. Bilhemia (bile in vascular system) could causepersistent deterioration of the liver function and confusion post procedure, which can be controlled after treating the fistula [18].The direction of blood flow and development of bilhemia and hemobilia is to a large extent influenced by the intravascular biliary duct pressure gradients. The pressures in healthy subjects are as follows. (1) hepatic artery $100 \mathrm{mmhg}$, (2) bile duct 10 to $15 \mathrm{mmhg}$, (3) portal vein $10 \mathrm{mmHg}$, and (4) hepatic vein 0 to 5 $\mathrm{mmHg}$ [18]. The pressure gradient between intrahepatic hollow structures can be affected by pathophysiological changes in various disease states, including cirrhosis and common bile duct obstruction. A supraphysiological biliary-venous pressure gradient $(>10 \mathrm{~mm} \mathrm{Hg})$ is needed to cause clinically significant bilhemia, which may happen in the presence of bile duct obstruction [18]. When pressure is $\leq 10 \mathrm{~mm} \mathrm{Hg}$, fistulas are likely to close spontaneously. When hepatic artery is involved, the highpressure system is more prone to persistence of fistula.

\section{Risk of hemobilia in patients with coagulation disorder}

Like any other invasive procedure, liver biopsy in patients with coagulation disorders including congenital bleeding disorders is at a risk of bleeding complications [22, 62]. These procedures however can be carried out safely in these patients, if they are under adequate cover of coagulation factors, in the periprocedure period [22]. There are differing opinions as to what constitutes an abnormal coagulation index that would determine a contraindication for liver biopsy. Most centers consider an international normalised ratio (INR) $>1.5$ and platelet count of $<50,000 / \mathrm{mm}$ as strong contraindication [61].

\section{The pathophysiology of Blood clot in Bile duct}

The blood does not normally clot within the bile duct because of fibrinolytic activity of bile [65]. However, sometimes the clot if formed, fails to lyse. Sandblom et al reported that when the hemorrhage is severe and rapid, the blood mixes with bile and forms mushy clot [65]. When hemobilia is minor and slow, the mixture does not result, but leads to blood flowing and settling at the bottom, where it forms a solid pure clot that forms cast of the lumen [65]. These pure clots remain stable, whereas mixed clots dissolve rapidly. Hence the fate of clots is related to the bile flow, with the dissolution of clot being rapid when flowing blood is exposed to bile $[65,66]$. The difference in surface tension between bile and blood could be another factor that could lead to stasis and clot formation [65].

\section{Presentation}

The classical presentation of hemobilia constitutes the Quincke's triad [2,9] and includes right upper quadrant pain, jaundice and acute gastrointestinal hemorrhage. This is reported in some series to be seen in $50 \%$ [38] to $63 \%$ of patients [39]; however it constituted $40 \%$ of the presentation in this review. The most frequent clinical finding is upper gastrointestinal bleeding (90\%); right upper quadrant pain $(70 \%)$ and jaundice $(60 \%)$ [9]. The mean interval is reported to be 5 to 6 days following the liver biopsy $[2,5,6,15,30,47]$. Hemobilia may present immediately $[11,21,57]$ or may be delayed up to a week or as late as 25days [39].The clinical manifestation of hemobilia is determined by the amount and speed of hemorrhage into the biliary tract $[1,6]$. Profuse hemobilia often causes severe symptoms with colicky pain and gastrointestinal hemorrhage $[65,66]$. However, often the bleeding is occult and lacks clinical significance as the blood inconspicuously flows into the intestine $[65,66]$. Blood clots in the gall bladder can mimic biliary colic or induce hemocholecystitis [20,21,45], while the blood clot in CBD may present with obstructive jaundice or complications of pancreatitis $[23,25,51,52]$ and cholangitis, similar to patients with choledocholothiasis $[1,12,24]$.

\section{Liver biopsy technique and its influence in causing hemobilia}

Liver biopsy is carried out by percutaneous transhepatic approach or by transjugular approach [11, 62]. Transjugualr approach is preferred in patients with severe coagulopathy, ascites, massive obesity or suspected vascular tumours $[4,11,62]$. Patients who require simultaneous vascular procedures (such as Venogram or Transjugular Intrahepatic Portosystematicshunt (TIPS) placement or when percutaneous biopsy has failed would be better served by a transjugular approach [62]. The procedure would involve, inserting a catheter into the hepatic vein through the right internal jugular vein, under fluoroscopy guidance. The liver biopsy forceps is then introduced to blindly obtain tissue. The drawback however is that the samples are smaller than those obtained by percutaneous approach $[4,62]$. It warrants several passes in order to obtain an adequate pathologic specimen with a reported success rate of $97 \%$ [4]. The complication rate is around $2 . \%$ and comprises mild (abdominal pain, neck hematoma transient Horner's syndrome) or more severe complications (hemobilia, gall bladder puncture, cardiac arrhythmias, pneumothorax, perforation of liver capsule, sampling of adjacent organs) [4]. Although ultra-sound guided biopsies are relatively safer, complications including hemobilia are reported $[9,13,25$, 48]. Complications of percutaneous liver biopsy are fortunately uncommon. Majority (96\%) of them appear in the first 24 hours and the remaining $4 \%$ usually have their clinical presentation within 10 days [50]. The presence of cirrhosis and neoplasm however increase the risk of complications [20]. In addition to the underlying disease, the risk of complications would also 
depend upon the coagulative status, the type and the diameter of biopsy needle used and the number of needle passes, despite being ultrasound guided $[6,9,62]$. US guidance however would reduce the risk of undesired puncture of surrounding organs.Major complications are higher in patients with focal lesion $(1.3 \%)$ compared to $0.3 \%$ in those without a focal lesion [7]. Hemobilia has also been reported following endoscopic ultrasound biopsy of hilar carcinoma through transdudoenal bulb approach [8] and also in those where the biopsy has been carried out laparoscopically $[19,32]$.

\section{Complications of hemobilia}

Several complications could occur as a consequence of blood in the biliary system [1].The blood clot could cause obstructive jaundice and may lead to acutecholecystitis [1,4,20,21], cholangitis [1,15] and acute pancreatitis [1, 9, 10, 12-14, 23], intra-abdominal fluid collection [4], persistent distension of gall bladder with gall bladder rupture [4], and acute gastric stress ulcers [1]. Blood clot could raise the intra biliary duct and pancreatic duct pressure leading to acute pancreatitis [1].The clot may disappear in few days time $[9,10]$ particularly in those who had interventions to drain the biliary system or it may persist longer [20]. In those where the clot in gall bladder persists, have been reported to form gallstones, few months later [35]. Percutaneous cholecystostomy could reduce the GB and biliary tract pressure [4]. Reducing the biliary tract pressure can prevent pancreatitis [1]. Arteriovenous fistula bleeding in the liver often results into an intra-hepatic hematoma and occasionally portal hypertension $[1,67]$. Lim et al have reported that percutaneous liver biopsy could result in arteriovenous fistula in 38\% (8/21) of cases after biopsy [67]. However vascular biliary injury noted in this review included arteriobiliary fistula (32.6\%), arterioportal fistula (36.9\%), pseudoaneurysm (21.7\%) and arterio venous biliary (trifistula) (4.3\%). The predominant complications noted in this review included pancreatitis (41.8\%), hemocholecystitis (21.8\%) and cholangitis (10.9\%).

\section{Investigations}

The bleeding through the papilla into the duodenum can be established by endoscopy in $33 \%$ [39] to $60 \%$ of the cases $[6,24,68]$. Ultrasound doppler could help in diagnosis of a pseudoaneurysm and would appear as a well circumscribed anechoic lesion with a turbulent flow $[1,6,24,55]$. Contrast enhanced CT scans can demonstrate hematoma, anatomical variations, pseudoaneurysm and cavitating lesions $[26,67]$. The appearance on US and CT varies depending on the rapidity and severity of bleeding. US and CT scan may note the hydropic gall bladder and surrounding edema compatible with cholecystitis [9, 22]. Intracholecystic bleeding typically appears as echogenic , non-acoustic polypoidal mass [22, 24]. The echotexture becomesa reticular stranding sludge, as clot lyses [24]. The border of the clot becomes concave and gall bladder appears to have polypoidal mass [24]. MRCP may reveal associated pancreatitis, cholecystolithiasis, cholangiectasis and abnormal signal indicating muddy stone or hematocele in CBD [1]. MRCP shows a negative magnetic signal in the biliary duct and gall bladder, corresponding to the clot in it [21]. If ERCP is performed, it could demonstrate the blood emerging from the papilla figure 1 and the intraluminal blood clot could be documented as filling defect figure $2[10,24]$. Digital subtraction angiogram (DSA) could detect the site of bleeding and fistula, when the rate of blood loss is $>0.5 \mathrm{ml} / \mathrm{min}$ [1]. A strong suspicion of hemobilia warrants an urgent angiography for its diagnostic and therapeutic value. This is in the form of an initial celiac axis angiography, combined with superior mesenteric arteriography, to determine a possible abnormal origin of an anomalous /accessory right hepatic artery from SMA in 20\% of the patients [6]. A selective right and left hepatic angiogram are likely to localize the bleeding better, particularly in the presence of pseudoaneurysm [6]. Unfortunately angiogram may appear normal in some patients in the absence of active bleeding or when there is no demonstrable lesion [6, 39]. In the event bleeding continues or recurs, a repeat angiogram is worthwhile and advisable [6].Angiography findings in hemobilia could include arterio-portal fistula, arteriobiliary fistula and pseudoaneurysm [24]. Arterio-portal fistula is reported to occur in 5\% of the cases and may remain asymptomatic [24].

\section{Treatment}

The main principle in management of hemobilia is resuscitation, control the source of bleeding and maintain the biliary patency $[9,39]$. Resuscitation with transfusion is carried out to obtain hemodynamic stabilitywhen hemorrhage is severe and rapid $[1,6,9,13,12]$. Any existing coagulopathies are simultaneously dealt with [22]. Nearly $50 \%$ of the patients will stop bleeding with the supportive measure of blood products $[9,22,24,37,42,55]$. The remaining patients would require therapeutic interventions, which would involve Transarterial Hepatic Artery Branch Embolization (TAE). TAE is most prudent in patients with pseudoaneurysm, arteriobiliary fistulas, or arterioportal fistula [1,69]. Superselective embolization is safer and more effective treatment for active bleeding. It is carried out using a microcatheter and is desirable as it minimizes spasm and avoids occlusion of the noninvolved arterial branches, particularly in liver transplant patients [69]. Hence, selective embolization as close as possible to the pseudoaneurysm or fistula is opted for,to limit the likelihood of both recurrence and liver necrosis $[48,69]$. The risk of inadvertent ischemic cholecystitis, hepatic artery main branches embolization, is also avoided [39].

In transplant patients, liver graft dysfunction and biliary ischemia are potential risks.

Embolisation could be carried out using gelatin sponge, autoallergy-sludged blood, spring orb, microcoils, polyvinyl alcohol particles and cyanoacrylate glue [1,6,24]. Coils induce thrombosis and hence for obvious reason may be ineffective in patients with gross coagulopathy [6]. Smaller pseudoaneurysm where coil placement may be difficult, may be effectively treated with cyanoacrylate glue as it conforms to the shape of the pseudoaneurysm,forms a cast instantly and is cheaper than microcolis [6]. Hypophysin is also reported to be commonly 
used [1].The success rate of arresting bleeding with TAE intervention is reported to range from 63\% [6], 94\% (16 out of 17) [6] to $100 \%$ [5]. In one of older series involving 11 cases hemobilia,transarterial embolization was successful in $63 \%$, with $18 \%$ having technical failure and I case $(9 \%)$ with ischemic cholecystitis [39]. Recent reports however indicate higher success rate $[5,6]$. Failure to arrest bleeding could be due to technical reasons or extensive collaterals [39].In the presence of extensive collaterals,embolization, distal and proximal to the pseudoaneurysm will be required [6]. Alternatively the pseudoaneurysm is occluded with microcoil or glue in addition to proximal occlusion with coils [6].Technical failure could also be due to a tortuous vessel, as in an allograft artery, post transplant [58] or due to intimal injury and spasm of the vessel, during manipulation of the catheter [60]. In very rare cases, it could be due to bleeding into peritoneal cavity of venous origin [11]. In one of the reports, persistent bleeding post TAE was localized to bleeding into the peritoneum from a feeding hepatic vein branch [11]. The bleeding, which was occurring through a subcaspular site, was effectively controlled by transjugular hepatic venogram and embolization [11]. In a post transplant patient where there

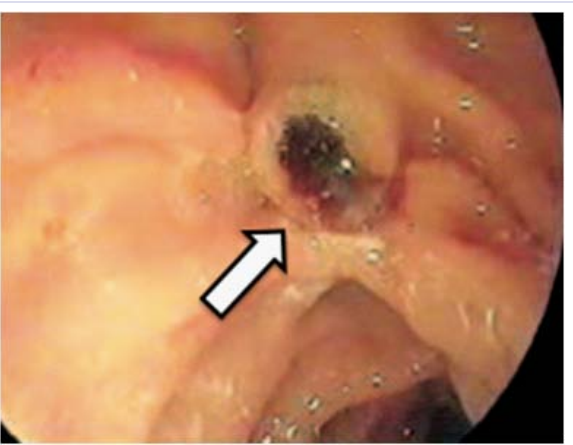

Figure 1: Blood Clot Seen at the Ampulla (arrow) in a Patient with Hemobilia.

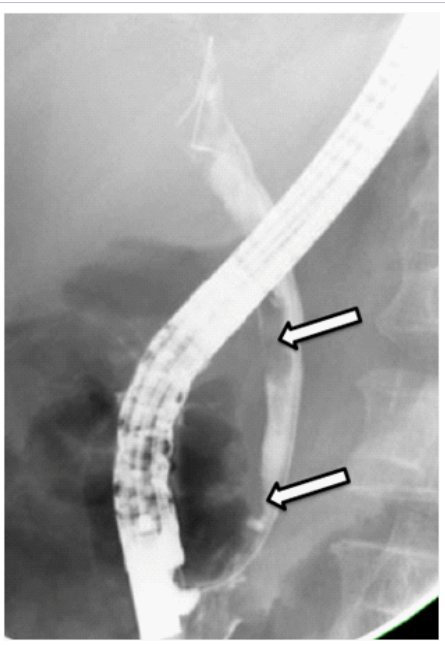

Figure 2: ERCP Revealing Multiple Clots in CBD as Filling Defects (arrows). is difficulty in achieving TAE because of technical difficulty in cannulating a tortuous allograft artery, the bleeding has been successfully dealt with by direct embolization of the aneurysm, under ultrasound guidance [58]. This review noted that TAE was carried out in $63 \%$ of the patients with $80 \%$ success in arresting the bleeding.

Having controlled the bleeding, the attention is then directed to a potential biliary tract obstruction, due to intraluminal clots. If the intraluminal clot does not dissolve and persist beyond 24 to 48 hours leading to progressive deterioration of liver function tests and dilatation of the bile duct (reflected by radiological imaging), then clot extraction and bile duct drainage would be required [4]. Biliary obstruction by clot may require ERCP sphincterotomy and balloon extraction of clots $[2,4,15,23]$. Recurrent clot formation after an initial successful clot extraction may warrant a repeat of the procedure [12]. Endoscopic nasobiliary drainage has also been used to decompress the biliary tract $[8,23]$. In addition, thrombolytic agents have been infused through a nasobiliary catheter to dissolve the biliary clots [70]. Routine placement of biliary stent in the presence of hemobilia is controversial. While some would stent the ampulla to prevent reaccumulation of clot [12], there are others who are concerned of stent forming a nidus which may act as matrix for thrombus attachment and worsening of obstruction [35, 37]. In liver transplant patients who develop hemobilia post liver biopsy, endoscopic approach may not be feasible because of anatomical distortion post bile duct reconstruction. In them, the clots can be dealt by transhepatic approach, to break up the clots progressively and increase its contact surface to flowing bile [71]. In patients with persistent distended gall bladder, a percutaneous transhepatic cholecystostomy could relieve the symptoms, particularly in patients with significant co-morbidities [4].

The role of surgery over the years has declined, after the advent of successful radiological intervention, in arresting bleeding in most of the cases[6, 24, 37].However, surgical intervention will be required when the above measures fail [6, 24].Emergency surgery to control major hemobilia is challenging and hazardous and should be avoided, as the results are poor [6]. Surgical intervention however is unavoidable in the absence of angiographic facility, failure of TAE or in the presence of hepatic sepsis [6]. In the absence of angiographic facility, every attempt should be made to transfer these patients to a center with these facilities. The principle of surgery is to control the bleeding vessels $[40,42]$, extraction of biliary clots with lavage and biliary tract drainage $[39,41]$. In the presence of liver necrosis or liver sepsis, a limited liver resection may be warranted. Post ERCP and sphincterotomy, reports suggest the disappearance of clot in GB, within a couple of weeks [24]. However in some cases, despite decompression of the biliary system by ERCP sphincterotomy, the clot in the gall bladder may persist, requiring cholecystectomy. This may deal with symptom related, both to acute cholecystitis or possible dyskinesia [21,35, 37].The timing of the cholecystectomy could either be immediate [20,35] or delayed [37], based on the existing symptoms and is carried out preferably laparoscopically. 
In addition to performing cholecystectomy, laparoscopic approach has been used to simultaneously carry out ligation of hepatic artery, in patients with persistent bleeding [30].

The reported mortality post iatrogenic hemobilia is around 10 to $12 \%$ and is mostly related to those who required surgical intervention [39]. In the recent years the success of angiographic embolization has significantly reduced the need for surgical intervention and hence the mortality. This review noted the mortality in $3.6 \%$ of the cases.

\section{Conclusions}

Hemobilia is a rare complication of liver biopsy, but is being noted with increasing frequency due its predominant role in establishing diagnosis in various liver pathology. The presentation could be variable but GI bleeding in the presence of jaundice and or pain post liver biopsy, should alert the clinician. Endoscopy and angiogram play a significant role, both as diagnostic and therapeutic tool. Off late, transarterial embolization arrests bleeding in majority of these patients. Role of surgical intervention is limited to those with complications of hemocholecystitis, failure of TAE in arresting bleeding and persisting clot induced obstructive jaundice, despite endoscopic intervention

\section{References}

1. Zhou HB. Hemobilia and other complications caused by percutaneous ultrasound-guided liver biopsy. World J Gastroenterol. 2014;20(13):3712-3715. doi: 10.3748/wjg.v20.i13.3712.

2. Qureshi MS, Iqbal $\mathrm{M}$, Butt MQ, Nomani AZ Iatrogenic post biopsy hemobilia with pseudoaneurysm. J Coll Physicians Surg Pak. 2014;24(11):865-867. doi: 11.2014/JCPSP.865867.

3. Zaleska-Dorobisz U, Lasecki M, Olchowy C Ugorski W, Garcarek J, Patkowski D, et al. Iatrogenic hemobilia in 10-year-old boy. Pol J Radiol. 2012;79:2792-82. doi: 10.12659/PJR.890410..

4. Plerhopies TA, Lau JN. Hemobilia from transjugular liver biopsy resulting in gall bladder rupture. Dig Dis Sci, 2013;58:630-33. doi: $10.1007 / \mathrm{s} 10620-012-2254-9$

5. Marynissen T, Maleux G, Heye S, Vaninbroukx J, Laleman W, Cassiman $\mathrm{D}$, et al. Transcatheter arterial embolization for iatrogenic hemobilia is a safe and effective procedure: case series and review of the literature. Eur J Gastroenterol Hepatol. 2012;24(8):905-909. doi: 10.1097/ MEG.0b013e328354ae1b.

6. Gandhi V, Doctor N, Marar S, Nagral A, Nagral S. Major hemobilia-experience from a specialist unit in a developing country.Trop Gastroenterol. 2011;32(3):214-218.

7. Howlett DC, Drinkwater KJ, Lawrence D, Barter S, Nicholson T. Findings of the UK national audit evaluating image-guided or image -assisted liver biopsy.Part 11. Minor and major complications and procedure-related mortality. Radiology. 2013;266(1):226-235. doi: 10.1148/radiol.12120224.

8. Kawakubo K, Isayama H, Takahara N, Yamamoto N, Kogure H, Sasaki $\mathrm{T}$, et al. Hemobilia as a rare complication after endoscopic ultrasoundguided fine-needle aspiration aspiration for hilar cholangiocarcinoma. Endoscopy. 2011;43 Suppl 2 UCTN:E334-335. doi: 10.1055/s-00301256783.
9. Egritas O, Dalgic B, Sendur HN. Cholecystitis, pancreatitis and hemobilia: a serious trilogy after liver biopsy. Pediatr Int. 2010;52(4):e215-217. doi: 10.1111/j.1442-200X.2010.03175.x.

10. Hendriks MP, Wanten GJ, Drenth JP. Management of hemobilia and pancreatitis after liver biopsy: a key role for endoscopic retrograde cholangiopancreaticography.Liver Transpl. 2009;15(11):1653-4. doi: 10.1002/lt.21807.

11. Koshy CG, Eapen CE, Lakshminarayan R. Transvenous embolization to treat uncontrolled hemobilia and peritoneal bleeding after transjugular liver biopsy. Cardiovasc Intervent Radiol. 2010;33(3):624-626. doi: 10.1007/s00270-009-9610-y.

12. Peña LR, Horn TL, Cross CB. Acute pancreatitis secondary to hemobilia after percutaneous liver biopsy. Therap Adv Gastroenterol. 2009;2(3):165-168. doi: 10.1177/1756283X08103654.

13. Wood B, Pearson N, Crisp M, Neese P, Pohl JF. Pseudoaneurysm formation after ultrasound-guided liver biopsy in an obese child. South Med J. 2009;102(4):432-434. doi: 10.1097/SMJ.0b013e31819bc364.

14.Li F, Mekeel KL, Eleid M, Harrison ME, Reddy KS, Moss AA, et al. Hemobilia and pancreatitis after liver transplant biopsy. Liver Transpl. 2009;15(3):350-351. doi: 10.1002/lt.21619.

15. Prata Martins F, Bonilha DR, Correia LP, Paulo Ferrari A. Obstructive jaundice caused by hemobilia after liver biopsy.Endoscopy. 2008;40 Suppl 2:E265-266. doi: 10.1055/s-2008-1077651.

16. Rogart JN, Aslanian HR .Massive hemobilia after transjugular liver biopsy treated endoscopically and angiographically. Clin Gastroenterol Hepatol. 2008;6(12):A30. doi: 10.1016/j. cgh.2008.06.013.

17. Gurakuqi GC, Stadlbauer V, Portugaller HR, Högenauer C, Trauner M, Stauber RE (2008). Fatal hemobilia resulting from an iatrogenic arteriobiliary fistula as a rare complication of transjugular liver biopsy. Eur J Gastroenterol Hepatol. 2008;20(1):83-86.

18. Bergmann OM, Sun S, Weydert J, Silverman WB (2007). Intrahepatic trifistula causing bilhemia and hemobilia resulting from transjugular liver biopsy in the setting of biliary tract obstruction. Gastrointest Endosc. 2007;66(4):848-850.

19. Dallal RM, Samuel G, Lord JL, Zaki R, Bailey L, Manzarbeitia C. Hemobilia: a rare complication after routine liver biopsy during gastric bypass. Surg Obes Relat Dis. 2007;3(1):91-93.

20.Edden $\mathrm{Y}$, St Hilaire $\mathrm{H}$, Benkov $\mathrm{K}$, Harris MT. Percutaneous liver biopsy complicated by hemobilia-associated acute cholecystitis.World J Gastroenterol. 2006;12(27):4435-6.

21. Albuquerque W, Arantes V, de Paula Farah K, Lambertucci JR. Acute pancreatitis and acute cholecystitis caused by hemobilia after percutaneous ultrasound-guided liver biopsy. Endoscopy. 2005;37(11):1159-1160.

22. Kruse-Jarres R, Leissinger CA. Haemobilia after transjugular liver biopsy in a patient with severe haemophilia. Haemophilia. 2005;11(6):642-643.

23. Nowak A, Hartleb M, Kohut M, Nowakowska-Duława E, PilchKowalczyk J. Haemobilia causing acute pancreatitis after percutaneous liver biopsy. J Hepatol. 2005;43(2):366.

24. Lin CL, Chang JJ, Lee TS, Lui KW, Yen CL. Gallbladder polyp as a manifestation of hemobilia caused by arterial-portal fistula after percutaneous liver biopsy: a case report. World J Gastroenterol. 2005;11(2):305-307. 
25. Hodgson RS, Taylor-Robinson SD, Jackson JE. Haematochezia in Crohn's disease caused by late-onset haemobilia following percutaneous liverbiopsy. Eur J Gastroenterol Hepatol. 2004;16(2):229-232.

26. Sharma R, Pancu DM. Hemobilia in the emergency department. J Emerg Med. 2004;6(2):201-203.

27. Asselah T, Condat B, Sibert A, Rivet P, Lebray P, Bernuau J. Haemobilia causing acute pancreatitis after percutaneous liver biopsy: diagnosis by magnetic resonance cholangiopancreatography. Eur J Gastroenterol Hepatol. 2001;13(7):877-879.

28. Coelho JC, Bonin EA, da Costa MA, da Cunha AG, Sartor MA . Acute cholecystitis secondary to hemobilia after percutaneous liver biopsy. Dig Surg. 2001;18(3):227-279.

29. Gómez-Valero JA, Sardi J, Vilaseca J, Pérez Lafuente M, Malagelada JR. Pancreatitis and haemobilia due to arterioportal fistula after percutaneous liver biopsy resolved by selective arterial embolization.

30. Eur J Gastroenterol Hepatol. 2001;13(6):727-730.

31. Gama-Odrigues J, Bresciani C, Seid VE. Videolaparoscopic management of percutaneous liver biopsy complications.Surg Laparosc Endosc Percutan Tech. 2001;11(2):134-138.

32. Rossi P, Sileri P, Gentileschi P, Sica GS, Forlini A, Stolfi VM, et al Percutaneous liver biopsy using an ultrasound-guided subcostal route. Dig Dis Sci. 2001;46(1):128-132.

33. Moehler M, Sanwald B, Kanzler S, Wanitschke R, Galle PR, HelmreichBecker I. Hemobilia after minilaparoscopic liver biopsy: a rare complication. Endoscopy. 2000;32(9):S60.

34. Eurvilaichit $C$. Iatrogenic hemobilia: management with transarterial embolization using gelfoamparticles. J Med Assoc Thai. 82(9):931937.

35. Jornod P, Wiesel PH, Pescatore P, Gonvers JJ (1999). Hemobilia, a rare cause of acute pancreatitis after percutaneous liver biopsy: diagnosis and treatment by endoscopic retrograde cholangiopancreatography. Am J Gastroenterol. 1999; 4(10):3051-3054.

36. Lee SL, Caruso DM. Acute cholecystitis secondary to hemobilia. J Laparoendosc Adv Surg Tech A. 1999(4):347-349.

37. Kim HJ, Shin JH, Lee YY, Lee JK, Yim BC, Park UT. Hemobilia causing acute biliary pancreatitis after percutaneous liver biopsy. Endoscopy. 1999;31(3):S18-19.

38. Kwauk ST, Cameron R, Burbridge B, Keith RG. Traumatic pseudoaneurysm of the hepatic artery after percutaneous liver biopsy and laparoscopic cholecystectomy in a patient with biliary cirrhosis: a case report. Can J Surg. 1998;41(4):316-320.

39. Richardson SC, Young TL. Liver biopsy-associated hemobilia treated conservatively.Tenn Med. 1998;91(4):141-142.

40. Dousset B, Sauvanet A, Bardou M, Legmann P, Vilgrain V, Belghiti J. Selective surgical indications for iatrogenic hemobilia. Surgery. 1997;121(1):37-41.

41. Lichtenstein DR, Kim D, Chopra S. Delayed massive hemobilia following percutaneous liver biopsy: treatment by embolotherapy. Am J Gastroenterol. 1992;87; 1833-1838.

42. Attiyeh FF, McSweeney J, Fortner JG. Hemobilia complicating needle liver biopsy. A case report with arteriographic demonstration. Radiology. 1976;118(3):559-560.
43. Ball TJ, Mutchnik MG, Cohen GM, Burrell M. Hemobilia following percutaneous liver biopsy. Gastroenterology. 1975;68(5 Pt 1):12971279.

44. Levinson JD, Olsen G, Terman JW, Cleaveland CR, Graham CP Jr, Breen KJ. Hemobilia secondary to percutaneous liver biopsy. Arch Intern Med. 1972;130(3):396-400.

45. Cox EF (1976). Hemobilia following percutaneous needle biopsy of liver. Arch Surg. 1967;95(2):198-201.

46. Cacho G, Abreu L, Calleja JL, Prados E, Albillos A, Chantar C, et al. Arterioportal fistula and hemobilia with associated acute cholecystitis: a complication of percutaneous liver biopsy. Hepatogastroenterology, 1996;43(10):1020-1023.

47.Sandblom P(1972). Hemobilia(biliary tract hemorrhage):History, pathology, diagnosis, treatment. Springfield,IL:Charles C Thomas.

48. Rossi P, Sileri P, Gentileschi P, Sica GS, Ercoli L, Coscarella G . Delayed symptomatic hemobilia after ultrasound-guided liver biopsy: a case report. Hepatogastroenterol. 2002;49: 1659-1662.

49. Hashimoto M, Akabane Y, HeiannaJ, Tate E, Ishiyama K, Nishii T, et al. Hepatic infarction following selective hepatic artery embolization with microcoils for iatrogenic biliary hemorrhage. Hepatol Res. 2004;30(1):42-50.

50. Sandblom P: Hemorrhage into the Biliary Tract Following Trauma"Traumatic Hemobilia."Surgery, 1948;24:571-586.

51.Piccinino F, Sagnelli E, Pasquale G, GiustiG(1986). Complications following percutaneous liver biopsy. A multicenter retrospective study on 68,276 biopsies. J Hepatol. 1986;2(2):165-173.

52. Machicao VI, Lukens FJ, Lange SM, Scolapio JS. Arterioportal fistula causing acute pancreatitis after liver biopsy. J ClinGastroenterol. 2002;34(4):481-484.

53. Sood A, Midha V, Kumar A, Sood N. Acute pancreatititis with hemobilia following percutaneous liver biopsy. Indian J Gastroenterol. 2002;21(2):81-82.

54.Grieco A, Bianco A, Pieri S, Costamagna G, Flammia G, Greco AV. Massive haemobilia after percutaneous liver biopsy in a patient with POEMS syndrome successfully treated by arterial embolization. Eur J Gastroenterol Hepatol. 1996;8(6):595-598.

55.Van Os EC, Petersen BT. Pancreatitis secondary to percutaneous liver biopsy-associated hemobilia. Am J Gastroenterol. 1996;91(3):577-80.

56. Jabbour N, Reyes J, Zajko A, Nour B, Tzakis AG, Starzl TE. Arterioportal fistula following liver biopsy. Dig Dis Sci, 1995;40(5):1041-1044.

57. deRibot X, Casellas F, Papo M, Vilaseca J, Moreiras M, Malagelada JR. Hemobilia causing acute pancreatitis after percutaneous liver biopsy. J Clin Gastroenterol. 1995;21(2):171-172.

58. Figueras J, Soubrane O, Pariente D, Devictor D, Houssin D. Fatal hemobilia after liver graft biopsy in a transplanted child. Gastroenterol Clin Biol. 1994;18(8-9):786-788.

59. Merhav H, Zajko AB, Dodd GD, Pinna A. Percutaneous transhepatic embolization of an intrahepatic pseudoaneurysm following liver biopsy in a liver transplant patient. TransplInt. 1993; 6(4):239-241.

60. Manzarbeitia C, Jonsson J, Rustgi V, Oyloe VK, Olson L, Hefter L. Management of hemobilia after liver biopsy in liver transplant recipients. Transplantation. 1993;56(6):1545-1547. 
61. Okazaki M, Ono H, Higashihara H, Koganemaru F, Nozaki Y, Hoashi T, et al. Angiographic management of massive hemobilia due to iatrogenic trauma. Gastrointest Radiol. 1991;16(3):205-214.

62. Grant A, Neuberger J. Guidelines on the use of liver biopsy in clinical practice. British Society of

63. Gastroenterology. Gut. 199;45(Suppl. 4): IV1-IV11.

64. Machado NO. Complications of liver biopsy-Risk factors, management and recommendations. INTECH. 2011; ISBN 978-953-307-644-7; doi:10.5772/21979.

65. Napolitano V, Cirocchi R, Spizzirri A, Cattorini L, La Mura F, Farinella E, et al. A severe case of hemobilia and biliary fistula following an open urgent cholecystectomy. W J EmergSurg. 2009;4:37. doi: 10.1186/1749-7922-4-37.

66. Poon RT, Tuen H, Yeung C. GI hemorrhage from fistula between right hepatic artery pseudoaneurysm and the duodenum secondary to acute cholecystitis. Gastro Endosc. 2000;51(4):491-493.

67. Sandblom P, Mirkovitch Y. Minor hemobilia: clinical significance and pathophysiological background. Ann Surg. 1979;190:254-264.

68. SandblomP. Iatrogenic hemobilia. Am J Surg. 1986;151(6):754-758.
69. Lee SJ, Lim JH, Lee WJ, Lim HK, Choo SW, Choo IW. Transient sub segmental hepatic parenchymal enhancement on dynamic CT: a sign of postbiopsyarterioportal shunt. J Comput Assist Tomogr. 1997;21: 355-360.

70. Curet P, Baumer R, Roche A, Grellet J, Mercadier M. Hepatic hemobilia of traumatic or iatrogenic origin: recent advances in diagnosis and therapy, review of the literature from 1976 to 1981 . World J Surg. 1984;8:2-8.

71.Saad WE, Davies MG, Rubens DJ, , Sahler LG, Patel NC, Lee DE, et al. Endoluminal management of arterio-portal fistulae in liver transplant recipients: A single center experience. Vasc Endovascular Surg. 2006; 40:451-459.

72. Moparty RK, Brown RD, Layden TJ, Chirravuri V, Wiley T, Venu RP. Dissolution of blood clots in the biliary ducts with a thrombolytic agent infused through nasobiliary catheter. Gastrointest Endosc. 2002;56:436-438.

73. Qin JJ, Xia YX, Lv L, Wang ZJ, Zhang F, Wang XH, et al. Successful disintegration and drainage of intracholedochal hematoma by percutaneous transhepatic intervention. World J Gastroenterol. 2012;18(47):7122-7126. doi: 10.3748/wjg.v18.i47.7122. 